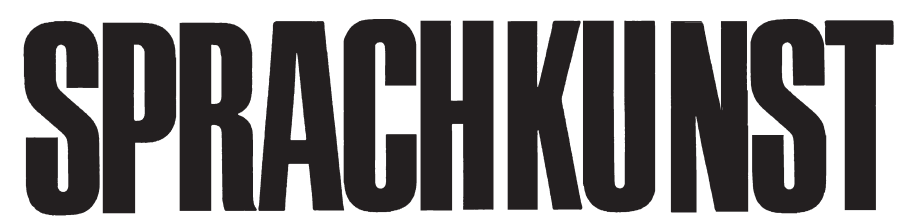

Beiträge

zur Literaturwissenschaft

Jahrgang XXXVIII/2007

2. Halbband 
Inhaltsverzeichnis

Aufsätze

Mahmoud Al-Ali

(Irbid)

Hartmut Vollmer (Paderborn)

Oswald Panagl (Salzburg)

Adolf Haslinger (Salzburg)

Wolfgang Kaltenbrunner (Maastricht)

Georg Schifko

(Wien)

Ute Mennecke

(Bonn)

Kari van Dijk

(Nijmegen)

Hans Höller

(Salzburg)

Walter Wagner

(Wien/Traun)

John Goodyear

(London)

Christian von Zimmermann

(Bern)

\section{Herbert Foltinek} (Wien)

Helga Mitterbauer

(Graz)
209 Der Passionsweg des Kassierers in Georg Kaisers Drama ,Von morgens bis mitternachtss.

219 Die Poetisierung stummer Traumbilder. Arthur Schnitzlers Pantomime `Der Schleier der Pierrette`.

243 Zerlines Genugtuung. Rezeptionssplitter von Mozarts ,Don Giovanni in Hermann Brochs `Die Schuldlosen`.

255 Dem Unerhörten zuhören. Zu Peter Handkes `Don Juan (erzählt von ihm selbst)،

263 Wissenschaft(skritik) bei Gottfried Benn und Primo Levi.

285 Zur Darstellung der Niam-Niam (Azande) und der Maori als Kannibalen und Kopftrophäensammler in Jules Vernes Romanen. Eine vergleichende imagologische Betrachtung.

295 VERZEICHNIS DER LITERATURWISSENSCHAFTLICHEN HABILITATIONEN AN ÖSTERREICHISCHEN UNIVERSITÄTEN

Berichte und Besprechungen

309 Claudia Resch, Trost im Angesicht des Todes. Frühe reformatorische Anleitungen zur Seelsorge an Kranken und Sterbenden (= Pietas Liturgica 15).

316 Arturo Larcati, Ingeborg Bachmanns Poetik.

321 Günther Stocker, Vom Bücherlesen. Zur Darstellung des Lesens in der deutschsprachigen Literatur seit 1945.

325 Stefan Hofer, Die Ökologie der Literatur. Eine systemtheoretische Annäherung. Mit einer Studie zu Werken Peter Handkes (= transcript).

330 Hans U. Werner, Soundscape-Dialog. Landschaften und Methoden des Hörens (= Edition Zuhören).

333 Schweizer Literaturgeschichte. Unter Mitarbeit von Claudia Brinker, Rémy Charbon, Regula Fuchs, Doris Jakubec, Ricarda Liver, Beatrice von Matt, Dominik Müller, Elsbeth Pulver, Clà Riatsch, Beatrice Sandberg, Antonio Stäuble, Stefan Bodo Würffel, Fred Zaugg herausgegeben von Peter Rusterholz und Andreas Solbach.

337 Renate Brosch, Short story - Textsorte und Leseerfahrung.

\section{Tagungsbericht:}

344 Gedächtnis und Erinnerung in Zentraleuropa. Jahrestagung des 'Literatur- und kulturwissenschaftlichen Komitees der Österreichischen und der Ungarischen Akademie der Wissenschaften`, 20.-22. September 2007, Wien.

\section{Diskussion:}

347 Zu: Franz K. Stanzel, Autobiographie. Wo ein Ich erzählt, ist immer Fiktion (Sprachkunst XXXVII/2006, 2. Halbband.)

Gedruckt mit Förderung des Bundesministeriums für Wissenschaft und Forschung in Wien.

Herausgegeben im Auftrag der Österreichischen Akademie der Wissenschaften, Kommission für Kulturwissenschaften und Theatergeschichte, von Herbert Foltinek und Hans Höller. Redaktion: Hermann Blume. Redaktionskomitee: Rudolf Baehr (Salzburg), Albert Berger (Klagenfurt), Georg Danek (Wien), Aage A Hansen-Löve (München), Friederike Hassauer (Wien), Wolfgang Riehle (Graz), Marie Luise Wandruszka (Bologna), Werner Welzig (Wien). 